\title{
Poly(3-hydroxyalkanoate)s Produced by Pseudomonas oleovorans Grown by Feeding Nonanoic and 10-Undecenoic Acids in Sequence
}

\author{
Young Baek Kim, ${ }^{\dagger}$ Young-Ha RheE, ${ }^{*}$ Robert W. LenZ, ${ }^{* *}$ and R. Clinton FulleR ${ }^{* * *}$ \\ Department of Polymer Engineering, PaiChai University, Daejon, Korea \\ * Department of Microbiology, Chungnam National University, Daejon, Korea \\ ** Polymer Science and Engineering Department, University of Massachusetts, Amherst, MA, U.S.A. \\ *** Biochemistry and Molecular Biology Department, University of Massachusetts, Amherst, MA, U.S.A.
}

(Received March 10, 1997)

\begin{abstract}
Pseudomonas oleovorans was grown on nonanoic acid and 10-undecenoic acid, which were fed in sequence. The poly(3-hydroxyalkanoate)s (PHAs) which were isolated from cells harvested at different growth times, were characterized by solvent fractionation, crosslinking ability, gel permeation chromatography, ${ }^{1} \mathrm{H}$ NMR spectroscopy, and differential scanning calorimetry. Two glass transitions were observed for the PHAs produced, and these corresponded to those of the PHAs produced from nonanoic acid [PHA(NA)] and 10-undecenoic acid [PHA(UA)] alone. The amounts of PHAs with repeating units produced from nonanoic acid remained constant while Pseudomonas oleovorans utilized UA for growth and PHA production. The molecular weight distributions of the PHAs obtained at different growth times were also constant. A crosslinking study showed that these polymers were mainly physical mixtures of PHA(NA) and PHA(UA). However, a fractionation study indicated that small amounts of the PHAs contained repeating units from both substrates, PHA(NA/UA).
\end{abstract} KEY WORDS PHA / Biopolymer / Biodegradable Polyester / Pseudomonas oleovorans /

Poly(3-hydroxyalkanoate) (PHA) production by Pseudomonas oleovorans has been investigated intensively as this microorganism produces polymers from a wide variety of carbon substrates, and the production of PHAs bearing functional groups such as $\mathrm{CN},{ }^{1}$ halogens, ${ }^{2-4}$ phenyl, ${ }^{5,6}$ phenoxy, ${ }^{7}$ olefin,,${ }^{8-10}$ and esters ${ }^{11}$ have been reported. In general, the PHAs produced by Pseudomonas oleovorans are elastomers with varying glass transition temperatures and melting temperatures ${ }^{12}$ depending on the nature of substituents in the side chains. PHAs containing $n$-alkyl side chains longer than an ethyl group melt below $60^{\circ} \mathrm{C}$ and posses glass transitions below $-20^{\circ} \mathrm{C}$, but PHAs containing phenyl and phenoxy groups have higher glass transition tempratures in the range of 0 to $10^{\circ} \mathrm{C}$.

Our recent results of PHA production with mixtures of nonanoic and 10-undecenoic acid are interesting. Pseudomonas oleovorans does not discriminate between these two carbon substrates in the production of PHAs, ${ }^{10}$ but cell and PHA yields from Pseudomonas oleovorans grown with the former alone are significantly lower than with the latter, and the lag phase observed in cell growth with 10-undecenoic acid is significantly longer than the lag phase with nonanoic acid.

PHAs produced by Pseudomonas oleovorans have been investigated intensively but little is known about the polymerization mechanism involved. The molecular weight distributions of the PHAs produced by Pseudomonas oleovorans are virtually identical regardless of carbon substrate, growth medium and growth time. The number average molecular weights are generally between 50000 and 70000 , with polydispersity indices between 2 and 3.

In this study we have investigated the PHAs produced by Pseudomonas oleovorans grown by feeding nonanoic acid, NA, and 10-undecenoic acid, UA, in sequence to determine whether it is possible to obtain

\footnotetext{
† To whom correspondence should be addressed.
}

block copolymers. NA and UA are ideal carbon substrates for this purpose because Pseudomonas oleovorans can utilize these substrates to support PHA production, but PHA production and growth of Pseudomonas oleovorans with each of these carbon substrates are significantly different. It was our primary concern, therefore, to understand how Pseudomonas oleovorans utilized UA, especially for polymerization, in the presence of PHA granules produced from NA.

\section{RESULTS AND DISCUSSION}

Figure 1 shows growth curves of Pseudomonas oleovorans obtained both by feeding NA and UA in sequence, and NA alone. The results from the sequential feeding experiments are listed in Table I. For fermentation A in Table I, UA was added when the rate of growth with NA started to decrease as indicated in Figure 1. For fermentation B, UA was added approximately $1 \mathrm{~h}$ after the gorwth with NA reached the stationary phase, so a small amount of NA might have been remaining in fermentation $\mathrm{A}$ when UA was added. In fermentation $\mathrm{B}$, the cells may have degraded the PHA produced from NA because there was no other available carbon source until UA was added. Indeed, the low PHA contents in cells harvested $1 \mathrm{~h}$ after UA was added strongly indicates that the PHA preservatives degraded when UA was added in fermentation B. Cell and PHA yields are higher for fermentation $B$ because the initial concentration of NA and the amount of UA added were higher in this case $(10 \mathrm{mmol}$ per liter $)$ than in fermentation A ( $5 \mathrm{mmol}$ per liter) as described in EXPERIMENTAL.

It was our main concern to understand how UA was utilized by Pseudomonas oleovorans for polymer production, and three possible pathways should be considered, namely: (1), repeating units produced from UA were added to the pre-existing PHA(NA) chain ends to produce block copolymers, PHA(NA-block-UA); (2), 
PHAs Produced from Sequential Feeding

Table I. Results from sequential feeding of nonanoic acid (NA) and 10-undecenoic acid (UA)

\begin{tabular}{|c|c|c|c|c|c|c|c|c|c|c|c|c|}
\hline \multirow{3}{*}{ Fermentation } & \multicolumn{4}{|c|}{ Dry cell weight $/ \mathrm{mg} \mathrm{L}^{-1}$} & \multicolumn{4}{|c|}{ PHA Content/dry cell \% } & \multicolumn{4}{|c|}{ PHA Yield/mg L ${ }^{-1}$} \\
\hline & \multicolumn{4}{|c|}{ Growth time $/ \mathrm{h}$} & \multicolumn{4}{|c|}{ Growth time ${ }^{a} / \mathrm{h}$} & \multicolumn{4}{|c|}{ Growth time $/ \mathrm{h}$} \\
\hline & 1 & 2 & 3 & 4 & 1 & 2 & 3 & 4 & 1 & 2 & 3 & 4 \\
\hline A & 316 & 523 & 565 & 616 & 32 & 34 & 33 & 35 & 101 & 175 & 185 & 215 \\
\hline B & 842 & 915 & 1178 & 1388 & 25 & 32 & 37 & 35 & 210 & 290 & 420 & 496 \\
\hline
\end{tabular}

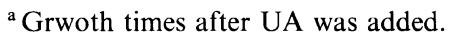

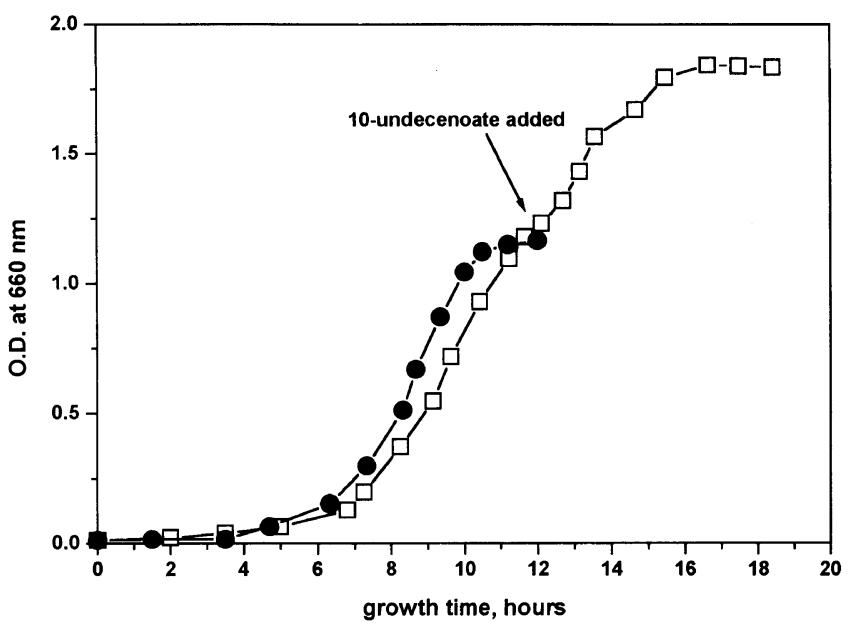

Figure 1. Growth curves of Pseudomonas oleovorans. (O), in a medium containing only $5 \mathrm{mM}$ of $\mathrm{NA}$; $(\square)$, in a medium containing $5 \mathrm{mM}$ of NA followed by addition of $5 \mathrm{mmol}$ of UA in a $1 \mathrm{~L}$ culture.

Pseudomonas oleovorans produced new polymer molecules from UA to yield physical mixtures of PHA(NA) and PHA(UA); (3), Pseudomonas oleovorans degraded pre-existing PHA(NA) and rebuilt a random copolymer containing repeating units from NA, PHA(NA-ranUA).

The DSC thermograms for PHAs isolated from cells harvested at different growth times, for a copolymer produced from an equimolar mixture of NA and UA, PHA(NA-ran-UND), and for the polymer from UA, PHA(UA), are shown in Figure 2. The thermograms reveal two glass transition temperautres in all PHAs isolated from cells harvested at different growth times, and the two glass transition temperatures correspond to those of the PHA obtained from each substrate alone. There is no significant change in melting temperatures in the different PHAs, but the DSC thermograms of PHA(UA) showed a melting endotherm at approximately $35^{\circ} \mathrm{C}$ when the sample was annealed at room temperature for several days. These results suggest that PHAs produced by Pseudomonas oleovorans grown by feeding NA and UA in sequence would be either PHA(NA-blockUA) or physical mixtures of PHA(NA) and PHA(UA).

The absolute amounts of repeating units produced from NA and UA in polymers isolated from cells harvested at different growth times were determined by ${ }^{1} \mathrm{H}$ NMR spectroscopy. A calibration curve was established using standard mixtures of PHA(NA) and PHA(UA). The mole fractions of repeating units containing unsaturated groups were calculated from their ${ }^{1} \mathrm{H}$ NMR spectra from the total areas of peaks from protons attached to unsaturated carbons and to $\beta$ -

Polym. J., Vol. 29, No. 11, 1997

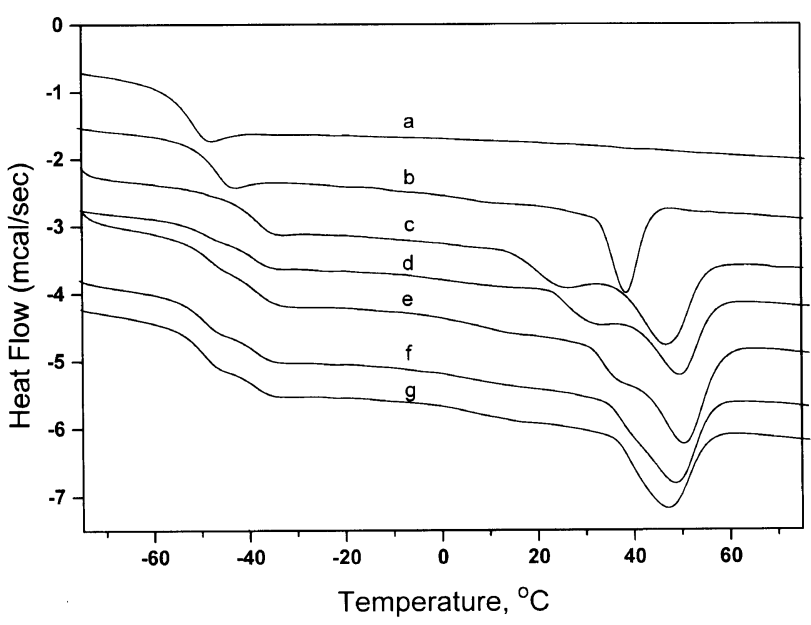

Figure 2. DSC thermograms of various PHAs. a, PHA(UA); b, PHA from an equimolar mixture of NA and UA; c, PHA(NA); d, e, f, g, PHAs isolated from cells growth for 1, 2, 3, $4 \mathrm{~h}$ after UA was added.

carbon. This calibration curve could be used to calculate the weight fraction of repeating units from UA because the composition of 3-hydroxyalkenoates units incorporated into PHAs was virtually identical regardless of growth conditions and growth time. ${ }^{10}$

Amounts of repeating units produced from NA and UA in the PHAs obtained at different growth times are shown in Figure 3. The plots for fermentation A in Figure 3 shows that the repeating units from NA increased until one hour after UA was added, indicating that NA still remained in the growth medium when UA was added. The plot for fermentation B in Figure 3 shows that the amount of repeating units from NA did not change after UA was added and that NA was depleted when UA was added in fermentation $\mathrm{B}$.

These results show that Pseudomonas oleovorans did not degrade the PHA(NA) stored in the cell while the PHA(UA) was produced. Figure 3 also shows that PHA production from UA by Pseudomonas oleovorans started shortly after this substrate was added indicating that Pseudomonas oleovorans did not discriminate between NA and UA for growth and PHA production.

The effect of nitrogen on degradation of PHA(NA) stored in the cell was examined by two step fermentation. Pseudomonas oleovorans was grown with NA under normal conditions and cells were harvested when the growth reached the stationary phase. The harvested cells were transferred into a nitrogen free medium containing UA. The growth curve, amounts of PHA(NA) and PHA(UA) produced at different growth times are shown in Figure 4. Figure 4 shows that there was approximately $9 \mathrm{~h}$ of lag phase for the growth with UA. 


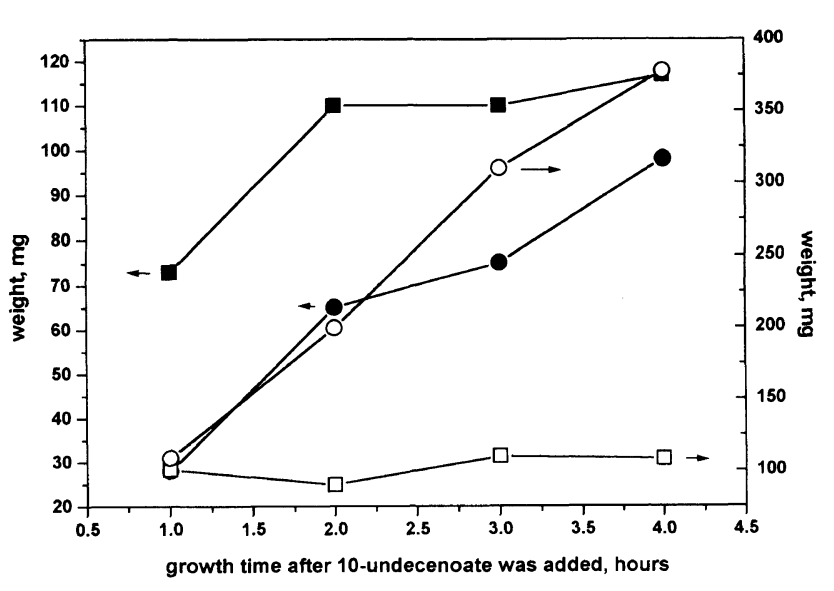

Figure 3. Amounts of repeating units in polymer products. ( $\square$ ) repeating units from NA in polymers produced from fermentation $\mathrm{A}$; (O), repeating units from UA in polymers produced from fermentation A; $(\square)$, repeating units from NA in polymers produced from fermentaiton B; $(O)$, repeating units from UA in polymers produced from fermentation $B$.

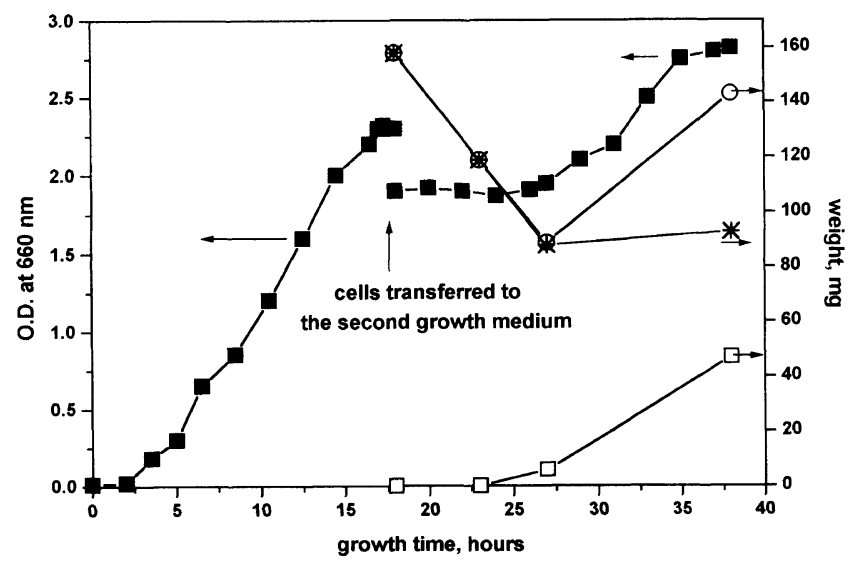

Figure 4. Fermentation results of two-step fermentation. (ם), O.D at $660 \mathrm{~nm}$; (O), weight of PHA obtained from $500 \mathrm{~mL}$ of culture; $(\square)$ weight of PHA(UA) in the PHA; $\left(^{*}\right)$, weight of PHA(NA) in the PHA.

Table II. Results of crosslinking-extraction experiments

\begin{tabular}{|c|c|c|c|c|}
\hline \multirow[t]{2}{*}{ Polymer } & \multirow{2}{*}{$\begin{array}{c}\begin{array}{l}\text { Weight of } \\
\text { polymer }^{\mathrm{a}}\end{array} \\
\mathrm{mg}\end{array}$} & \multirow{2}{*}{$\begin{array}{l}\text { Weight of unsaturated } \\
\text { repeating units in } \\
\text { the polymer }\end{array}$} & \multirow{2}{*}{$\begin{array}{c}\begin{array}{c}\text { Weight of extracted } \\
\text { polymer }\end{array} \\
\mathrm{mg}\end{array}$} & \multirow{2}{*}{$\begin{array}{l}\text { mol\% of unsaturated repeating } \\
\text { units in extracted polymer }\end{array}$} \\
\hline & & & & \\
\hline$A^{b}$ & 117 & 58 & 55 & 1 \\
\hline $\mathrm{B}^{\mathrm{c}}$ & 117 & 34 & 79 & 2 \\
\hline
\end{tabular}

${ }^{a}$ Weight of polymer used for crosslinking experiment. ${ }^{b}$ A control prepared by mixing the same amount of PHA(NA) and PHA(UA). ${ }^{c}$ The PHA isolated from cells harvested $1 \mathrm{~h}$ after 10 -undecenoate was added in fermentation A. ${ }^{\mathrm{d}}$ The PHA isolated from cells harvested $2 \mathrm{~h}$ after 10 -undecenoate was added in fermentation $\mathrm{B}$.

There was not noticeable consumption of oxygen but approximately $30 \mathrm{wt} \%$ of PHA(NA) disappeared during this lag phase. The amount of PHA(NA) remained constant after the cell started to grow and produced PHA(UA). Similar results were obtained from a two step fermentation carried out using nitrogen rich media. In summary, these results show that Pseudomonas oleovorans did not degrade PHA(NA) already produced as long as another carbon substrate was utilized for growth and PHA production.

If polymers produced were block copolymers of repeating units from NA and UA, PHA(NA-block-UA), the molecular weight of the PHA would increase with growth time after UA was added. However, molecular weight distributions of polymers obtained at different growth times were identical as determined by gel permeation chromatography. The number average molecular weights and polydispersity indices were approximately 60000 and 2.7 , respectively. The constant molecular weight distribution indicates that polymers obtained in this study were not PHA(NA-block-UA).

Crosslinking experiment was carried out by reacting the PHA products with $\mathrm{BH}_{3} \cdot \mathrm{BH}_{3}$ crosslinks only PHA molecules that contain carbon-carbon double bonds. The crosslinked product was washed with chloroform and the amount of soluble fraction was measured. If PHA(NA-block-UA) was produced, the amount of soluble fraction would be very small. The results from the crosslinking-extraction experiments listed in Table II show that the amount of extracted polymer was very close to the amount of PHA(NA) in the uncrosslinked polymer, and the mole percent of unsaturated unit in the extracted polymer was less than $2 \mathrm{~mol} \%$. These results clearly show that the PHAs obtained from sequential feeding were not PHA(NA-block-UA) but were physical mixtures of PHA(NA) and PHA(UA) instead. Neverethless, the fractionation study indicated these polymers might contain very small amounts of PHAs that contain repeating units from both NA and UA.

Fractionation of the PHA was carried out by dissolving the polymers in $n$-hexane at $30^{\circ} \mathrm{C}$. PHA(NA) dissolves in $n$-hexane while PHA(UA) forms a viscous slurry at $30^{\circ} \mathrm{C}$. Two layers formed with $n$-hexane was added to mixtures of PHA(NA) and PHA(UA). The upper layer was solution of PHA(NA) dissolved in $n$-hexane and the bottom layer was a slurry of PHA(UA) swollen with $n$ hexane.

Mixtures of known amounts of PHA(NA) and PHA(UA) were prepared as control and fractionations were carried out using these standard mixtures and PHAs isolated at different growth times. The weights and compositions of polymers recovered from the two layers are listed in Table III. Table III shows that upper layers of PHAs obtained by sequential feeding contained significantly more unsaturated units than upper layers of standard mixtures. These results suggest that a small 
PHAs Produced from Sequential Feeding

Table III. Fractionation results of various PHA samples

\begin{tabular}{|c|c|c|c|c|c|}
\hline Sample & $\begin{array}{l}\text { wt } \% \text { of unsat'd unit } \\
\text { in the polymer }\end{array}$ & $\begin{array}{l}\text { wt } \% \text { of polymer } \\
\text { recovered from the } \\
\text { upper layer }{ }^{\mathbf{a}}\end{array}$ & $\begin{array}{l}\mathrm{wt} \% \text { of polymer } \\
\text { recovered from the } \\
\text { bottom layer }\end{array}$ & $\begin{array}{l}\mathrm{wt} \% \text { of unsat'd unit } \\
\text { in the polymer } \\
\text { recovered from the } \\
\text { upper layer }\end{array}$ & $\begin{array}{l}\text { wt } \% \text { of unsat'd unit } \\
\text { in the polymer } \\
\text { recovered from the } \\
\text { bottom layer }\end{array}$ \\
\hline $\begin{array}{c}1: 1 \\
\text { Mixture }^{\mathrm{b}}\end{array}$ & 50.6 & 50.2 & 49.8 & 12.3 & 87.3 \\
\hline $\begin{array}{c}1: 2 \\
\text { Mixture }^{b}\end{array}$ & 33.7 & 68.0 & 32.0 & 11.8 & 85.5 \\
\hline $\begin{array}{c}2: 1 \\
\text { Mixture }^{\mathrm{b}}\end{array}$ & 66.3 & 37.2 & 62.8 & 20.0 & 88.8 \\
\hline $\mathrm{A}-1^{\mathrm{c}}$ & 28.8 & 78.6 & 21.4 & 22.8 & 72.3 \\
\hline$A-2^{c}$ & 36.5 & 75.9 & 24.1 & 28.7 & 76.7 \\
\hline$A-4^{c}$ & 52.3 & 69.2 & 30.8 & 34.4 & 80.0 \\
\hline B- $1^{\mathrm{c}}$ & 51.8 & 58.2 & 41.2 & 24.1 & 88.3 \\
\hline B- $2^{\mathrm{c}}$ & 69.5 & 44.1 & 55.9 & 34.8 & 88.2 \\
\hline B- $3^{\mathrm{C}}$ & 70 & 29.5 & 70.5 & 46.2 & 88.4 \\
\hline B- $4^{\mathrm{c}}$ & 77.8 & 24.0 & 76.0 & 38.4 & 89.1 \\
\hline
\end{tabular}

a weight of polymer recovered

weight of polymer dissolved in hexane

${ }^{\mathrm{c}}$ A- $n$, B- $n$, results for cells harvested $n$ hours after 10 -undecenoate was added from fermentation A and B, respectively.

fraction of PHA molecules obtained by sequential feeding might contain repeating units from both NA and UA, but these polymers would be produced either by pathway (1) to yield a block copolymer or by pathway (3) to yield a random copolymer.

\section{SUMMARY}

Results obtained in this study show that Pseudomonas oleovorans does not degrade a PHA already in the cell when there is another carbon source available. This behavior is different from that of Alcaligenes eutrophus which was reported to degrade poly(3-hydroxybutyrate) when exposed to new carbon substrates that support production of poly(3-hydroxybutyrate-co-3-hydroxyvalerate). ${ }^{13}$ Considering that Pseudomonas oleovorans did not discriminate between NA and UA in PHA production, and that PHA(UA) was produced as soon as UA was available in the one-step fermentation, production of PHA(NA-block-UA) was most likely prohibited by factors that are responsible for constant molecular weight distribution. A probable explanation is that the chain ends of PHA molecules are buried inside the polymer granules so that they are not available to chain growth reaction. Therefore, understanding the mechanism that results in constant molecular weight distribution would enable one to biosynthesize PHA(NAblcok-UA).

\section{EXPERIMENTAL}

\section{Materials}

Tetrahydrofuran (THF) was freshly distilled from sodium benzophenon ketyl. Chloroform was dried over sodium carbonate and then distilled and stored over molecular sieve $(3 \AA)$. All other chemicals used in this study were used as purchased.

\section{Biosynthesis of PHA}

Pseudomonas oleovorans was grown under aerobic conditions as $3 \mathrm{~L}$ cultures using a fermenter equipped with temperature controller. Agitation speed was $200 \mathrm{rpm}$ and air was supplied at a rate of $0.5 \mathrm{~L} \mathrm{~min}^{-1}$. Growth medium was prepared and treated as described elsewhere. ${ }^{14}$ The initial concentrations of NA were $5 \mathrm{mM}$ and $10 \mathrm{mM}$ for fermentation $\mathrm{A}$ and fermentation $\mathrm{B}$, respectively. The amount of added UA was $5 \mathrm{mmol}$ and $10 \mathrm{mmol}$ per one liter of growth medium for fermentation $\mathrm{A}$ and fermentation $\mathrm{B}$, respectively. The growth was monitored by measuring the optical density at $660 \mathrm{~nm}$. For fermentation A, UA was added when the rate of optical density increase started to decrease. Fermentation B was carried out monitoring the concentration of dissolved oxygen. The concentration of dissolved oxygen decreased to zero as soon as the growth reached the exponential growth phase. The concentration of dissolved oxygen returned to the value of lag phase when stationary growth phase was reached. In fermentation B, UA was added to the culture approximately one hour after the concentration of dissolved oxygen returned to the value of lag phase. Five hundred milliliters of the culture were harvested at different growth times and the residue was harvested when the growth rate started to decrease. Cells were freeze-dried and polymers were extracted into hot chloroform using a Soxhlet extractor. Extracts were dissolved in a small amount of chloroform and precipitated by dropping into vigorously stirred methanol. Precipitation was repeated three times to obtain final products.

Two-step fermentations were carried out by transferring cells grown in a nitrogen rich medium containing NA to either a nitrogen rich medium containing UA or a nitrogen free medium containing UA. The cells grown with NA were harvested when the growth reached the stationary growth phase. The cells were washed with sterilized distilled water and then transferred to the second medium. The growth was monitored by measuring optical density and the concentration of dissolved oxygen. Five hundred milliliters of the culture were harvested at different growth times and growth was stopped when the growth reached the stationary phase or when the concentration of dissolved oxygen resumed 
that of stationary phase.

\section{PHA Composition}

${ }^{1} \mathrm{H}$ NMR was used to determine the amount of repeating units from UA. Standard mixtures of PHA(NA) and PHA(UA) were prepared and ${ }^{1} \mathrm{H}$ NMR spectra of these mixtures were recorded. Mole percent of PHA(UA) in these mixtures were calculated from integrated areas of peaks from protons attached to unsaturated carbons and the proton attached to the carbon located at $\beta$ position from the ester group in the main chain. The mole percent calculated from ${ }^{1} \mathrm{H}$ NMR measurement and weight percent were used to establish a standard curve. Samples for ${ }^{1} \mathrm{H}$ NMR measurement were prepared in a concentration of $15 \mathrm{mg} \mathrm{mL}^{-1}$ to minimize deviation caused by different concentration.

\section{Fractionation}

Mixtures of known amounts of PHA(NA) and PHA(UA) were prepared as control. $n$-Hexane was add to control and PHAs isolated from cells grown for different growth times and the mixture was shaken vigorously above $30^{\circ} \mathrm{C}$. One and half milliliters of $n$-hexane was used for $100 \mathrm{mg}$ of polymer. The mixture was allowed to stand in an oven maintained at $30^{\circ} \mathrm{C}$ for $30 \mathrm{~min}$. The upper layer, PHA(NA) solution in $n$-hexane was taken using a disposable pippet and $n$ hexane in both layers was evaporated at room temperature. For complete dryness, $n$-hexane was evaporated under reduced pressure overnight at room temperature. Products obtained were weighed and ${ }^{1} \mathrm{H}$ NMR spectra were taken. Concentrations of polymers for NMR analysis were approximately $15 \mathrm{mg} \mathrm{mL}^{-1}$.

\section{Crosslinking of $\mathrm{PHAs}$ with $\mathrm{BH}_{3}$}

Crosslinking was carried out using mixtures of known amounts of PHA(NA) and PHA(UA) as well as PHAs obtained at different growth time. Hundred milligrams of polymers were dissolved in $2 \mathrm{~mL}$ of $1: 1(\mathrm{v} / \mathrm{v})$ mixture of chloroform and THF. To this solution $0.5 \mathrm{~mL}$ of $1 \mathrm{M}$ $\mathrm{BH}_{3}$-THF solution was added. Gel was produced as soon as $\mathrm{BH}_{3}$ was added. The gel was allowed to stand for $30 \mathrm{~min}$ and few drops of distilled water was added. Excess chloroform was added and the mixture was stirred vigorouly using a vortex mixer. The products were filtered through a glass tube clogged with cotton. The solbule products were recovered by evaporating solvents. The soluble fraction was weighed and analyzed using ${ }^{1} \mathrm{H}$
NMR.

\section{PHA Characterization}

Differential scanning calorimeter measurement was carried out using a Perkin-Elmer DSC 7. The temprature was scanned from -100 to $180^{\circ} \mathrm{C}$ at a ramp of $20^{\circ} \mathrm{C}$ $\min ^{-1}$. NMR spectra were recorded using a Bruker AM 300 or a Bruker AMX 500.

Molecular weights of PHAs were determined using a gel permeation chromatography system equipped with Waters 6000 solvent delivery system, RI detector, and U6K injector. A linear Ultrastyragel, $10^{3} \AA$, and $10^{4} \AA$ Waters Styragel columns were used. A standard curve was established with standard polystyrene samples. Samples were prepared in concentrations of approximately $0.3 \mathrm{wt} \%$ in chloroform. Approximately $100 \mu \mathrm{L}$ was injected and chloroform was used as the eluent.

Acknowledgment. This work was supported by the Basic Science Research Institute Program (Project No. 95-4430), Ministry of Education, Korea.

\section{REFERENCES}

1. R. W. Lenz, Y. B. Kim, and R. C. Fuller, FEMS Microbiol. Rev., 103, 207 (1992).

2. Y. Doi and C. Abe, Macromolecules, 23, 3705 (1990).

3. C. Abe, Y. Taima, Y. Nakamura, and Y. Doi, J. Chem. Soc., Polym. Commun., 31, 404 (1990).

4. Y. B. Kim, R. W. Lenz, and R. C. Fuller, Macromolecules, 25, 1852 (1992).

5. K. Fritzsche, R. W. Lenz, and R. C. Fuller, Macromol. Chem., 191, 1957 (1990).

6. Y. B. Kim, R. W. Lenz, and R. C. Fuller, Macromolecules, 24, $5256(1991)$

7. Y. B. Kim, Y. H. Rhee, S. H. Han, G. S. Heo, and J. S. Kim, Macromolecules, 29, 3432 (1996).

8. G. Langeveen, G. W. Huisman, P. Preusting, P. Ketelaar, G. Eggink, and B. Witholt, Appl. Environ. Microbiol., 54, 2924 (1988).

9. K. Fritzsche, R. W. Lenz, and R. C. Fuller, Int. J. Biol. Macromol., 12, 85 (1990).

10. Y. B. Kim, R. W. Lenz, and R. C. Fuller, J. Polym. Sci., Part A, 33, 1367 (1995).

11. Y. B. Kim, Ph.D. Thesis, University of Massachusetts at Amherst, 1991.

12. K. D. Gagnon, R. W. Lenz, R. J. Farris, and R. C. Fuller, Macromolecules, 25, 3723 (1992).

13. Y. Doi, A. Segawa, Y. Kawaguchi, and M. Kunioka, FEMS Microbiol. Lett., 67, 165 (1990).

14. R. A. Gross, C. DeMello, R. W. Lenz, H. Brandl, and R. C. Fuller, Macromolecules, 22, 1106 (1989). 